\title{
Youth Enrichment through Sport: The YES Ghana Model
}

\author{
William D. Ramos \\ Indiana University, Bloomington, IN \\ Sarah J. Young \\ Indiana University, Bloomington, IN
}

\begin{abstract}
It's no secret that youth today in the United States (U.S.) and around the world face mounting public health challenges in their daily lives. Factors such as obesity, alcohol use, and engagement in sexual activity continue to be top of mind for public health professionals, government officials, parents, and most recently; parks and recreation professionals. When administered in an appropriate and professional manner, these programs can help youth develop key life skills such as self-confidence, self-efficacy, and empathy. In addition, if parks and recreation professionals also take on the responsibility of positively impacting public health issues, these same programs can also create change in population well-being. This paper outlines one such program that we believe can serve as a model for using recreational sports programs as the mechanism to intervene in negative youth health behaviors.

The Youth Enrichment through Sport (YES) Ghana program was a two-year endeavor funded through the Bureau of Education and Cultural Affairs within the U.S. Department of State to encourage a cultural exchange and provide a sustainable and effective program model to impact public health for the youth in the Cape Coast region of Ghana, Africa. Although developed and implemented thousands of miles away, lessons learned can easily be applied to many regions of the United States toward the development and administration of a recreational sports program. Such programs would have the potential to make an impact on the public health population.
\end{abstract}

Keywords: Recreational, Sport, Youth, Public, Health, Intervention

William D. Ramos is an assistant professor with the Department of Recreation, Park, and Tourism Studies at Indiana University, Bloomington. Sarah J. Young is an associate professor with the Department of Recreation, Park, and Tourism Studies at Indiana University, Bloomington. Please send all correspondence to William D. Ramos, wramos@indiana.edu.

Recreation, Parks, and Tourism in Public Health I.I (2017).

Copyright $\odot$ Trustees of Indiana University. doi: Io.2979/rptph.I.I.08 


\section{Youth Enrichment through Sport: The YES Ghana Model}

Youth in the United States and around the world face mounting pressures to give in to negative health behaviors. Traditional public health programs, although showing some promise, generally lack the ability to bring youth together in an environment that is amenable to communicating and educating them on the major issues (Geidne, Quennerstedt, \& Eriksson, 20I3; Mays, DePadilla, Thompson, Kushner, \& Windle, 20I0). Benefits from youth involvement in recreational sports is well documented including factors such as: (a) development of self-esteem, (b) development of self-confidence, and (c) ability to function in team settings (Kaufman \& Wolff, 20Io; Nathan, Kemp, Bunde-Birouste, MacKenzie, Evers, \& Shwe, 2013). Not only does involvement in organized sport make a difference but the purposeful design of the program and thoughtful delivery can also produce desirable outcomes. The sport activity itself is the mechanism, or "hook", that allows organizers to draw youth in through a love of play, and to then achieve desired outcomes. Our program model was established on the fundamental belief that recreational sports can be used to bring youth together and influence factors related to key areas of concern for an array of public health issues including: (a) substance abuse, (b) sexual behaviors, and (c) physical inactivity.

The purpose of the Youth Enrichment through Sport (YES) program design was to provide a positive and engaging environment for youth to come together through sport with purposefully designed and delivered public health messaging woven into the sport program enabling students to make healthier life choices. This article is presented to provide an overview of the program, its goals, implementation, and lessons learned about using sport-for-development for youth ro-r6 years of age.

\section{Program Background and Structure}

The YES program was designed to reach underserved girls and boys at junior high and senior high school levels, especially marginalized at-risk youth, living in Apewosika Township near Cape Coast, Ghana (see Figure I). Funded with a grant from the U.S. Department of State-Bureau of Education and Cultural Affairs, the program involved a two-way exchange of adult volunteer youth leaders coming to the U.S. to be trained to facilitate after-school recreational sport programs. YES representatives from Indiana University (IU) also traveled to Cape Coast to monitor and support the programs throughout the life of the grant.

The goals of the program were to not only use selected team sports as a way to improve physical activity and fitness levels, but also to communicate 
Figure I: Map of Ghana and Cape Coast Region.
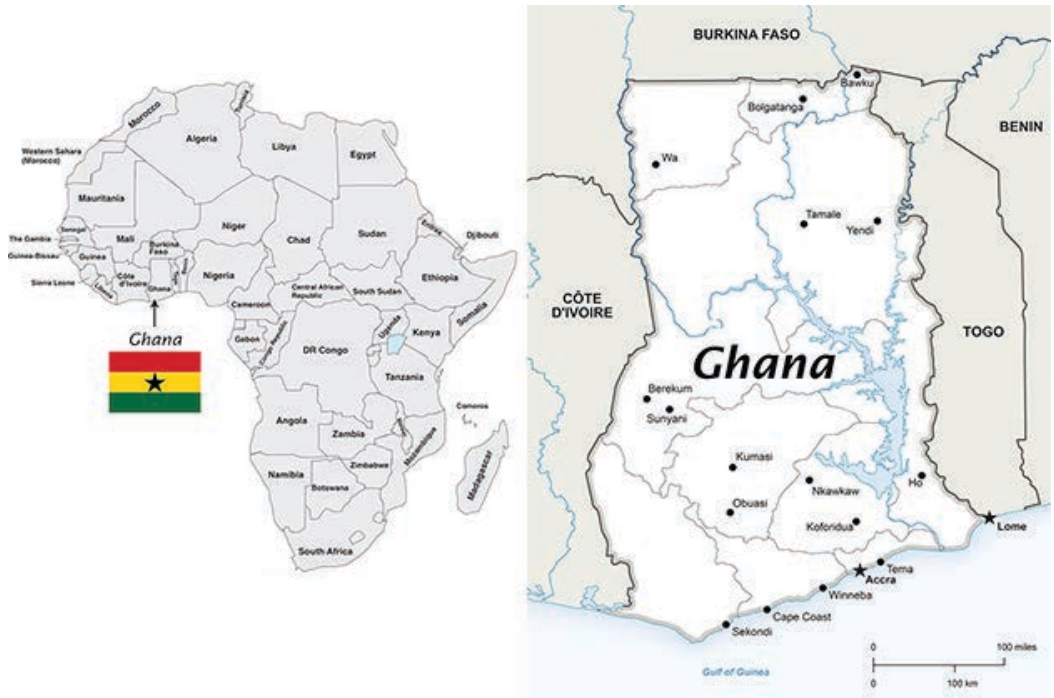

and educate youth on the importance of healthy living. Program goals were achieved by increasing the professional capacity of adults from Ghana involved in youth sport programming delivery, as well as increasing their proficiency in sport instruction (i.e., soccer: boys and girls, basketball: boys and volleyball: girls). These after-school programs were geared toward the average to below average skilled participant, or non-elite athlete.

Ghana was selected for this project due to the fact that it is a democratic country with a stable government presenting an enabling environment for collaborative projects (BBC News, 20I7). With a population of over 25 million people inhabited by 75 ethnic groups, Ghana has been able to avoid the kind of ethnic conflict frequently found in other African countries (Ghana Embassy, 2017). With over $95 \%$ of its children attending school, Ghana currently has one of the highest enrollment rates for all of Africa (The Forum for Education Reform, 2013). Because a goal of this program was to extend sport opportunities to youth through after-school recreational sports opportunities, the schools provided a logical access point to our target population.

Cape Coast is located in the South Central Region of Ghana and has access to some of the best schools in the country, yet is still considered rural with an economy primarily dominated by farming and fishing. People living in urban areas of Ghana account for only $37 \%$ of the population, with the remaining twothirds of the population living in rural areas (Ghana Embassy, 20I7). Within Cape Coast, the Apewosika Township was selected because it was identified as a locale where many youth did not have access to organized sport programs, and there was an expressed interest from school teachers to help implement the program. 


\section{Local Public Health Issues}

Alcohol and drug abuse. The influence of regional culture on drug use by children in Africa is evident. Specifically, the literature on current alcohol and drug abuse and misuse in-country indicates that children who begin to drink at an early age have a higher risk of developing family problems in adulthood (Obeng, 20II; DeWit, Adlaf, Offord, et al., 2000).

Concerning youth access to alcohol in African countries in general, and in Ghana in particular, it is very easy for youth to drink alcohol (Presley, Meilman, \& Lyerla, I995; Koriah, 2006). There are a variety of alcoholic drinks which are inexpensive making them affordable for youths. Furthermore, home-brewed beverages tend to have high alcohol content. For example, akpeteshie (a kind of gin) has 50\% alcohol by volume; palm wine has $5.2 \%$ alcohol by volume, and pito (millet wine) has $3 \%$ alcohol by volume (Centers for Disease Control and Prevention, 2003). The availability of such cheap and high alcoholic-content drinks puts youths at risk for early experimentation and abuse.

HIV/AIDS. The prevalence of HIV/AIDS among men and women aged I5 to 49 is estimated to be about $\mathrm{I} .5 \%$, though the prevalence rate in the region varies significantly by area and is as high as $9.6 \%$ in some urban areas (Takyi, 2003). Recent data indicates increases in prevalence among adolescent women and men, I5-19 years old. Although nearly all women and men in Ghana are aware of HIV/AIDS, knowledge about HIV/AIDS and safer sex behaviors have been slower to change. A 2008 report suggested that about $72 \%$ of I $^{-2}-24$ year old women and about two-thirds of $15-24$ year old men are considered to have insufficient knowledge of HIV (Ghana AIDS Commission, 20I4). Major goals in HIV prevention efforts in Ghana include: (a) delaying the age of sexual debut, (b) encouraging sexually active men and women to limit their number of sexual partners, (c) and increasing condom use. Data suggest that only about I in 4 men and less than one-half of women report using a condom during high risk sexual behavior (Heath, I995).

A consequence of the HIV/AIDS epidemic in Ghana is that approximately I $4.5 \%$ of orphan children-or about $\mathrm{I} 60,000$ children-have been orphaned specifically due to one or both of their parents dying from AIDS (Heath, I995). In particular, orphaned youths are at a higher risk of dropping out of school programs due to HIV/AIDS related stigma, for financial reasons, or for staying home to care for sick relatives. Furthermore, HIV/AIDS stigma remains a serious barrier for people who themselves are infected with HIV/AIDS or whose families members are HIV-positive, which poses a particular threat to children who have HIV-positive family members and who may be ostracized from participation in school or sports activities. HIV/AIDS stigma also prevents people from seeking testing and treatment (even though treatment 
for HIV/AIDS is typically free) and from accessing condoms (which tend to cost money). Our Time Out for Healthy Living sessions taught information that was consistent with the national strategies to reduce HIV/AIDS in Ghana (Ghana AIDS Commission, 20I4).

\section{Program Frameworks}

The eight features of positive youth development settings (Eccles, \& Gootman, 2002) provided a useful framework for guiding the training programs offered to adult leaders through the YES-Ghana program. In addition, the program was grounded in the covenants of the Easy Play Model that focuses on developing opportunities for play that promote an inclusive and supportive environment for less skilled participants through a low structure, flexible, and dynamic grouping design (Lu \& Steele, 20I4). The ultimate goal of the YES-Ghana program was to provide junior and senior high school youth the opportunity to build relationships with key adult leaders who, through their training and skill sets, could play a role in resilience enhancement through participants' experiences. Supportive individuals, including peers as well as adults, were necessary components in fostering resilience, and were stressed as a key component of the grant initiative. A premise of the YES-Ghana sports initiative was that youth sport coaches play a monumental role in the development of youth and successful youth sport programs (Green \& Chalip, I997; Seefeldt \& Ewing, I996). As a result, technical, sport-specific skills and competencies related to the delivery of effective youth sport programs were provided in training workshop for YES-Ghana coaches and volunteer leaders along with the broad range of coaching principles and positive youth development concepts that were integrated into the sport activities.

\section{Program Themes}

YES Ghana included three thematic areas to drive program development and delivery:

I. Provide an after-school recreational youth sports program for junior high and senior high school aged youth living in rural Apewosika Township, Ghana. This goal captured a sport engagement theme by focusing on the physical activity needs of underserved youth with average to below average sport skills living in rural areas without regular access to sport facilities. Youth learned sport skills and rules in soccer, basketball and volleyball. Training and educational sessions were provided to a selected group of adults from Apewosika Township who desired to lead youth to 
participate in an after-school recreational sport program. These adult leaders received instruction for coaching, sport program management, and sport officiating so that they could create sustainable youth sport programs in their communities for years to come.

2. Provide health education for coaches and youth through "Time Out for Healthy Living" sessions. While involving children in sport provides them with increasing levels of physical activity and fitness leading to greater quality of life, sport participation can also be the key to educating youth about the exigencies of greater health risks such as substance abuse and the prevention/ management of non-communicable or infectious diseases. This goal met the sport and health theme of the initiative by providing educational sessions for both adult leaders and participating youth.

3. Provide "Time Out for Healthy Living" sessions focused upon leadership development, teamwork, responsibility, and self-discipline as an avenue by which to educate youth to become better citizens positioned to make positive contributions to their communities. In meeting the third and final goal of the project, sport for social change, "Time Out" sessions also were provided on tolerance and inclusion, principles of teamwork, sportsmanship and character development, leadership skills, and self-discipline. Educational sessions for both adult leaders and youth were designed to mitigate school dropout rates among Ghana youth, assist them in forging a common bond, develop as responsible citizens, and consequently minimize youth violence.

\section{Program Phases}

Phase one-Partnership, Volunteers, and Context. The initial phase of the project involved formalizing partnerships, gaining a real-life context in locations we would be working, and soliciting volunteers to be trained to deliver the programs. Fliers, social media, and other public service announcements were made in Cape Coast to attract volunteers. Interested individuals completed an application which was reviewed by a team of YES representatives and embassy officials, with top finalists interviewed to determine the cohort traveling to the U.S. While in Cape Coast for interviews the project team also toured the region and area schools to get a better sense of the assets available as well as to make connections and negotiate agreements with partners on the ground. These relationships proved to be crucial for the success of the program due to the vast distance between the project team and the host site. Main collaborators in Cape Coast came primarily from faculty and graduate students at the University of Cape Coast School of Health, Physical Education, and Recreation (HPER). 
One of the most impactful messages coming out of the first phase was a resounding call from local village leaders that whatever program we started had to be sustainable in design. A tribal chief in particular noted that although he appreciated many of the programs that non-profit organizations have brought to the area in the past, most were short lived and not realistic to maintain after the organization left. The chief noted that this type of help can actually create a sense of despair and failure for the community. With this powerful message in hand, we returned to the U.S. with a commitment to re-visit our current plan and make adjustments as needed to provide a sustainable program model.

Early on it also became apparent that relationships built on-site would be key in determining the success of the program. Due to physical and cultural differences, it was imperative to have "boots on the ground" or, individuals who could facilitate communication and handle the myriad of details involved in the travel exchange. For the YES program these partners emerged from our relationship with faculty at the University of Cape Coast, School of HPER.

Phase two-Training Workshop and Cultural Exchange. The second phase involved hosting a I2-day workshop in the U.S. for the I6 selected volunteer adult leaders from Ghana, with a focus on:

- Organizing, planning, managing, and programming youth recreational sports programs;

- Coaching methods and strategies for boys' and girls' soccer, boys' basketball and girls' volleyball;

- Instruction on rules and officiating of youth soccer, basketball, and volleyball.

- Health education sessions focused upon substance abuse, reduction of HIV-related stigma, and the prevention and management of noncommunicable or infectious diseases, such as sexually transmissible infections (STIs), including HIV.

- Educational sessions focused upon tolerance and inclusion, principles of teamwork, sportsmanship and character development, leadership skills, and self-discipline.

- Educational and hands-on session about sports injuries, treatment, and warm-up exercises for success and injury prevention.

At the core of YES-Ghana was its "value-added" clinics and workshops presented by experts in (I) leadership training; (2) health education; (3) sportsmanship at the NCAA; and (4) sports medicine with IU Athletic Training experts. These workshop sessions were reinforced by site visits to university and community facilities.

All of the workshops presented volunteer coaches with the skills and materials they need to hold "Time Out" sessions during their sport training 
programs. Our expert trainers crafted short, well-designed 20- to 30-minute presentations for our audience to take back to Cape Coast and implement. These presentations addressed specific topics such as "working well with others," "communicating for success," "respect for people who are different," "making healthy choices," etc. The clinics and workshops actually showed the adults how to conduct short "Time Out for Healthy Living" sessions for youth. This part of YES-Ghana training made the after-school recreational sport program a platform for educating youth in leadership skills, character development, sportsmanship, respect for diversity, and tolerance that could carry over into the students' private and public life.

Phase 3-Site Visits to Check for Action. Two site visits took place to ensure that programs were implemented upon our group's return to Ghana. The first visit was taken within one month after the team returned to implement the program before the end of the school year. We felt although there was only about two months remaining in the school year that it was important not to wait until the fall for fear of losing momentum and the excitement that leaders felt to share what they had learned during the U.S. workshop. This short term start was also seen as a good pilot period, with the summer, then seen as a time to refine and organize for a full school year roll out in the fall. The second site visit occurred in the fall about three months after the start of the new school year with a similar focus as the first.

Phase 4-On-Site Training and Evaluation. For the final official phase of the study, a team of sport and public health professionals traveled to Ghana to monitor and evaluate the program and to provide additional sport and health education clinics. The theme of this final visit to Cape Coast and each of the after-school programs was to "train the trainer" in an attempt to facilitate the sustainability of the programs. New schools interested in starting a YES program were invited, as well as additional volunteers, recruited by current volunteers running existing programs. This training plan included site visits to each of the original schools for on-site training through modeling for volunteers, as well as performing a final assessment to gain a snapshot of the current public health situation regarding physical activity, substance abuse, and sexual behavior.

Evaluation. To evaluate the YES program several data collection points were conducted throughout the exchanges and site visits. One point of assessment came from the training workshop provided to Ghana volunteers in the U.S. This piece was crucial for helping to identify areas in which volunteers felt there was a disconnect from the information presented and any realities in the context of their specific situations. The survey captured a level of satisfaction using five point Likert scales ( $\mathrm{I}=$ poor, $5=$ excellent) using $\mathrm{I} 6$ questions clustered in to three focus areas including: (a) sport sessions $(M=4.69)$, (b) time out for healthy living sessions $(M=4.27)$, (c) field trip experiences $(M=4.64)$, and (d) overall experience summary $(M=4.56)$. The overall mean from the scale data was 4.54 with each sub area scoring a minimum of 4.0 with the exception 
of two areas which were: (a) length of the program $(M=2.3)$, and (b) and two items referencing the quality of the food provided $(M=2.73$ and 3.88).

A second assessment was made once programs were established and underway to examine what was working, what was not working, and to provide an opportunity to brainstorm solutions for challenges encountered. This information was collected through program journals kept by the lead volunteers for each program site. Comments received provided valuable insight into successes taking place and challenges to be addressed.

The final evaluation piece involved the administration of a survey based on the CDC's Youth Risk Behavior Surveillance System (Eaton, et al., 2015) and the Godin Leisure-Time Exercise Questionnaire (Godin \& Shephard, 1997) to obtain a snapshot of youth behavior for participants related to substance abuse, sexual activity, and physical activity. Information from this survey revealed that youth participating in the program had lower negative behavior scores related to the constructs measured as compared to national census data for Ghanaian youth. This was discovered when looking into behaviors related to: (a) physical inactivity, (b) smoking/tobacco use, (c) alcohol abuse, (c) substance abuse, and (d) risky sexual activity.

\section{Lessons Learned}

Although this program was implemented in another region of the world, much was learned that is relevant in applying the same type of program in the United States. The following is an overview of lessons learned:

I. Program sustainability must be a conversation very early on in the planning process and should include a complete understanding of the local customs and culture. Many times organizations go into an area of need to provide valuable services that are not designed to continue after the initial investment is made. Using a community participatory approach and gaining commitments from community leaders to invest in the long term viability is key. It is also equally important to design the program with sustainability in mind. For us this included a "training the trainer" process by which the leaders we trained were taught who to engage and how to train others, this kept the volunteer base advancing. Another factor is teaching people to be resourceful with materials on-hand and to involve local skilled tradesmen to help build permanent sports equipment. For example, our team employed the skills of an architect who provided easy to follow design plans on how to build basketball backboards and rims out of locally available items.

2. We learned that the public health messages did not have to have a separate moment in the program design, but instead could be literally integrated into the sports skills or game portions of the program. This 
was taught to us by one of the volunteers who took the information we provided and incorporated the content of the messages directly into the activities through singing, chanting, and soccer ball-handling drills.

3. If you are coming into a community from the outside, you may never really understand the cultural context of the people you are trying to serve. Make your best attempts to immerse yourself in the community, be willing to let go of pre-conceived ideals, and beware of trying to assert your personal beliefs and context to an area that simply functions differently than where you come from. The most success and sustainability will come from programs that match their communities and make sense to the population being served.

4. Use of the Easy Play Model which was employed as a foundational delivery model provides an even playing field in the delivery of recreational sports (Lu \& Steele, 20I4). Students seemed to gravitate to the idea of a less competitive atmosphere and one based more on inclusion and cooperation. An interesting phenomena we noticed in Ghana was that some students actually chose not to try out for a "varsity" programs once they experienced the easy play model of the YES program because it allowed them to play regardless of ability.

5. Activity based programs have the capacity to truly change lives and communities. Over the two year span of implementing the YES program, we saw engaged, happy, and active youths. Through the results of our survey and anecdotal comments from volunteers, teachers, and parents, a positive impact was felt in the community.

6. Be flexible, ready, and willing to make changes on the fly as needed. Even the best planned program can miss a step or simply miss the mark due to intervening variables. Be creative, be resourceful, and create an avenue so you can listen to the people in the program to know when changes need to be made.

7. Building strategic relationships is paramount to a successful program. Selecting key contacts who are both capable and invested provides invaluable "boots on the ground" to help with on-going communication and immediate support. These relationships need to be bi-directional in nature with benefits and respect on both sides. Program sponsors should make sure not to over-tax partners and take the time to understand political and cultural system at play.

\section{Conclusion}

Having the opportunity to develop and implement a program of this type overseas has in turn allowed us to realize that many of the concepts, successes, 
and challenges would be the same in a U.S. context. For many U.S. rural and urban areas with at-risk youth, a lack of organized sports opportunities, and failure to create an even playing field to allow for all abilities and skill levels, a program such as YES-Ghana can provide a framework for impactful and sustainable youth interventions.

Through recreational sports we have the innate ability to engage youth by using activities they already view as positive and create an audience for important messaging on making healthier choices. By using our skills in leisure and recreation to not only attract youth but also to evoke our unique ability to organize and deliver positive experiences, we found ourselves in a unique position to use our experience and resources to reach a greater good by taking on a public health agenda.

Where did we see the similarities and carry over to what was accomplished a world away? We saw that youth love to play, that youth are facing growing pressures in negative areas of public health, that community volunteers will step up to take stewardship to make a difference, that there are challenges regarding intrapersonal, interpersonal, and structural barriers that can be overcome with creative thought and open minds, and that something purposefully designed to be sustainable can have longevity.

The profession is calling those of us in the field of parks and recreation to take ownership of our role in public health. We see this in the charges by respected governing bodies such as the National Recreation and Park Association, NIRSA: Leaders in Collegiate Recreation, America Public Health Association, and World Health Organization. The example of the YES-Ghana program provides a way to integrate what we do best by making a positive public health impact.

\section{REFERENCES}

BBC News. (2017, January 19). Ghana country profile. Available at: http://www.bbc.com /news/world-africa-I3433790

Centers for Disease Control and Prevention. (2003). Ten strategies for promoting physical activity, healthy eating, and a tobacco-free lifestyle through school health programs. Available at: http://www.cdc.gov/healthyyouth/publications/pdf/ten_strategies.pdf. Accessed January $23,2013$.

DeWit, D. J., Adlaf, E. M., Offord, D.R., et al. (2000). Age at first alcohol use: a risk factor for the development of alcohol disorders. American Journal of Psychiatry, 157(5):745-750.

Eaton, D. K., Kann, L., Kinchen, S., Shanklin, S., Ross, J., Hawkins, J., .. \& Lim, C. (2010). Youth risk behavior surveillance-United States, 2009. Morbidity and mortality weekly report. Surveillance summaries (Washington, DC: 2002), 59(5):I-I42.

Eccles, J. S., \& Gootman, J. A. (2002). Features of positive developmental settings. Community programs to promote youth development, 86-II8. 
Geidne, S., Quennerstedt, M., \& Eriksson, C. (2013). The youth sports club as a health promoting setting: An integrative review of research. Scandinavian Journal of Public Health, 41:269-283.

Ghana AIDS Commission. (2012, March). Ghana Country AIDS Progress Report. Available at: http://www.unaids.org/en/dataanalysis/knowyourresponse/country progressreports $/ 2012 \mathrm{C}$

Ghana AIDS Commission, (20I4, March 3I). Country AIDS Response Progress ReportGhana. Available at: http://files.unaids.org/en/dataanalysis/knowyourresponse /countryprogressreports/20I4countries/GHA_narrative_report_20I4.pdf

Ghana Embassy (2017). About Ghana. Available at: http://www.ghanaembassy.org /index.php?page=about-ghana

Godin, G., \& Shephard, R. J. (1997). Godin leisure-time exercise questionnaire. Medicine and Science in Sports and Exercise, 29(6):36-38.

Green, B.C., \& Chalip, L. (1997). Enduring involvement in youth soccer: The socialization of parent and child. Journal of Leisure Research, 29(I):6I-77.

Heath, D. B. (1995). An anthropological view of alcohol and culture in international perspective (pp. 328-334). In D. B. Heath (Ed.), International Handbook on Alcohol and Culture. Westport, CT: Greenwood Press.

Kaufman, P. \& Wolff, E. A. (2010). Playing and protesting: Sport as a vehicle for social change. Journal of Sport and Social Issues, 34(2):154-175.

Koriah, C. J. (2006). Alcohol and empire: "illicit" gin prohibition and control in colonial Eastern Nigeria. African Economic History, 31:III-I34.

Lu, C., \& Steele, K. (2014). Characteristics and conceptual framework of the Easy-Play Model.European Physical Education Review, 20(2):229-243.

Mays, D., DePadilla, L., Thompson, N. J., Kushner, H. I., \& Windle, M. (2010). Sports participation and problem alcohol use: A multi-wave national sample of adolescents. American Journal of Preventive Medicine, 38(5):49I-498.

Nathan, S., Kemp, L., Bunde-Birouste, A., MacKenzie, J., Evers, C., \& Shwe, T. A. (2013). "We wouldn't of made friends if we didn't come to Football United": The impacts of a football program on young people's peer, prosocial, and crosscultural relationships. BMC Public Health, 13:399-415. Obeng, C. S. (2011). Parental perspectives on alcohol use among school-aged children in Ghana. International Electronic Journal of Health Education, 14:59-67.

Presley, C. A., Meilman, P. W., \& Lyerla, R. (1995). Alcohol and Drugs on American College Campuses: Use, Consequences, and Perceptions of the Campus Environment, Volume II:I990-92. Carbondale, IL: Core Institute, Southern Illinois University.

Takyi, B. K. (2003). Religion and women's health in Ghana: Insights into HIV/AIDS preventive and protective behavior. Social science \& medicine, 56(6):I22I-I234.

The Forum for Education Reform. (2013, December 19). State of Education in Ghana. Available at: https://www.modernghana.com/news/509540/state-of-education-in -ghana.html 